\title{
Study Protocol to Investigate the Efficacy of Participation in Qi-Gong by Breast Cancer Survivors*
}

\author{
Shing Yee Lee ${ }^{1 \#}$, Siew Yim Loh" ${ }^{1 \#}$, Liam Murray ${ }^{2 \#}$ \\ ${ }^{1}$ Department of Rehabilitation Medicine, Faculty of Medicine, University of Malaya, Kuala Lumpur, Malaysia; ${ }^{2}$ Centre for Public \\ Health, School of Medicine, Dentistry and Biomedical Sciences, Queens University of Belfast, Belfast, Ireland. \\ E-mail: siewyimloh@yahoo.com
}

Received September $5^{\text {th }}, 2011$; revised November $11^{\text {th }}, 2011$; accepted November $23^{\text {rd }}, 2011$.

\begin{abstract}
Background: Physical activity improves quality of life and reduces the risks of breast cancer up to 30 - 40 percent. Qi-Gong is a form of exercise widely acknowledged by Asian survivors as health promoting, despite a lack of research evidence. This study aimed to investigate the efficacy of Qi-Gong on the Quality of Life (QOL) of survivors. Methods/ Design: A total of 114 women who had been treated for stage I or II breast cancer at least 12 months previously were randomly assigned to supervised aerobic exercise $(n=38)$, Qi-Gong exercise $(n=38)$, or usual care $(n=38)$. Supervised 1.5 hour exercise sessions took place three times per week for 8 weeks (face to face-once a week and home follow up twice a week). Outcomes included QoL (FACT-G), Functional Assessment of Cancer Therapy-Breast (FACT-B)], distress [Depression Anxiety Stress Scale (DASS)] assessed at baseline and at the 8-week follow-up. Secondary measures included exercise Self Efficacy Scale (ESS), Functional Assessment of Cancer Therapy-Fatigue (FACT-F) and exercise behavior (log) Two way (group $\times$ time) repeated measures ANOVA will examine differences between groups over time. Discussion: This research will provide insights on the efficacy of a 8 weeks structured Qi-Gong program designed for quality of life. The details of the Qi-Gong and the placebo (line dancing) over 8 weeks are delineated, with the study protocol for researcher's replication. The result of the study can contribute towards understanding how Qi-Gong over other physical activity can be used for self managing physical health for people with breast cancer, an increasingly acknowledged chronic condition. Conclusion: Women with breast cancer need to start engaging in physically healthyactive living on a day-to-day basis for prevention of recurrence, mortality and wellbeing. However, many requires professional support with a structured intervention. This design of this study provides a protocol for 8 weeks Qi-Gong with implications on healthy survivorship, and a possible reduction on healthcare costs related to long-term effects and co morbidities that can be prevented via physical activity.
\end{abstract}

Keywords: Cancer, Qi-Gong, Physical Activity, Protocol

\section{Introduction}

Breast Cancer is the most common cancer for women above age 20 for all races in Malaysia. 31.1\% of all cancer in year 2003-2005 is breast cancer. A woman in Malaysia is predicted to have 1 over 20 chance of getting breast cancer in her lifetime, with Chinese has the highest possibility (1:16), follow by Indian (1:17) and Malay $(1: 28)[1]$. Advances in breast cancer detection and treatments have increased women's chances of surviving [2].

\footnotetext{
*Competing interests: The author(s) declared that they have no competing interests.

\#Authors' Contribution: S. Y. Loh, S. Y. Lee, and L. Murray conceptualized the study. S. Y. Loh and S. Y. Lee contributed to the write up.
}

The increasing cancer survivors faces unique challenges from their disease and treatments, including risk for recurrent cancer, other chronic diseases, and persistent adverse effects on physical functioning and quality of life. Interventions for women with breast cancer must also be unique to the health needs of women [3], covering broader issues like participation and healthy-living experiences [4]. This is because 1) participation in everyday occupations exerts positive impact on health and wellbeing [5], and 2) women with breast cancer are living for indefinite period due to better treatment. Current media attention and funding is timely for breast cancer disease, a highly prevalent disease in women worldwide [6]. Unfortunately, 
even with this attention, medical care for women with breast cancer continues to focus narrowly on detection and survival. In many developing countries, care for women with breast cancer has neglected the less visible psychosocial aspects [7] which ironically, appears to be the overarching goal for women with breast cancer in the developed world [8]. Emphasis on cancer survivorship and lifestyle factors like active lifestyle is grossly neglected. In this study, we adopt the definition of "cancer survivor" purported by the National Coalition for Cancer Survivorship, i.e., from the time of diagnosis until the end of life (http://www.canceradvocacy.org (Accessed April 13, 2009).

Prompt, continuing education and support are fundamental basic needs of cancer patients [9], but, in most developing countries like Malaysia, education and emotional support are hardly essential parts of the clinical practice guidelines. This neglect is serious since women with breast cancer require intense and prolonged treatments [10], and many of the cancer treatment's aftereffects are not identified yet.

Evidence is convincing that physical activity engagement and active life styles reduces the risks of breast cancer mortality. As with many chronic conditions, effective management of significant morbidity from illness and its treatment which reduces QOL have taken on increasing importance [11]. In short, although these tasks such as managing emotions are usually addressed in psychosocial groups, the other important aspects on management of illness and daily roles [12] may not be fully integrated. Lifestyle changes are common after a cancer diagnosis, and cancer survivors report changing to increase wellbeing, maintain health, and prevent recurrence [13]. Physical activity has been found to enhance QOL in breast cancer survivors [14-17]. Physical activity can improve physical fitness, reduce fatigue, increase functioning, and enhance overall QOL in breast cancer survivors both during after treatment(s) [18]. Physical activity is associated to about $25 \%$ to $30 \%$ decrease in risk ( $n=62$ studies) and $83 \%$ studies with positive findings reported a doseresponse effects [19]. Beneficial exercise-induced changes in cancer patients has also been reported in 26 studies [20].

In the Nurses' Health Study (NHS, $\mathrm{n}=280$ ) with a 8 years median follow-up; women who exercised the equivalent of 3 to 5 hours per week of walking had half the risk of dying of breast cancer (relative risk $=0.50$, $95 \%$ confidence interval 0.38 to 0.84 ), with no evidence for increased benefit for greater exercise [21]. Some literature reviews show that being physically active is more beneficial amongst postmenopausal women breast cancer survivors [19,22]. US Centers for Disease Control and Prevention has recommended for 2.5 hours per week of moderate aerobic activity for all adults [23] (Physical
Activity for Everyone). One of the physical activities that cancer survivors engaged in as complementory therapy is Qi-Gong. Qi-Gong shows promise in ameliorating cancer side effects and preliminary studies report immediate improvement without side effects, even recording complete remission in patients who engaged in ongoing practice of it [24]. The author reported that Qi-Gong is safe and effective in improving QOL, fatigue, mood status and reducing symptoms, side-effects and inflammation in cancer patients. Others findings postulate that Qi-Gong is able to increase pain threshold combined with a relaxation effect, improve microcirculatory function and improve of immune function [25].

In Malaysia, Qi-Gong is seen as a therapeutic form of physical activity for cancer patients, even though there is little research evidence on the effectiveness of Qi-Gong with cancer patient in Malaysia. Thus, this study will evaluate the effectiveness of Qi-Gong and its relationship with QOL of breast cancer survivors at post-treatment phase. Enabling women to live a healthy life after breast cancer is an important agenda in cancer survivorship and is in line with the goal of occupational therapy. Thus this paper aim to present the study protocol of Qigong in contrast to another physical activity (i.e. Line dancing) and delineates the protocol of 8 weeks sessions for QiGong and Line Dancing so that other researcher can replicate the entire study.

\section{Research Design and Methods}

\subsection{Aim of the Paper}

This paper present the outcome of a planning team to developed a 8 week structured Qi-Gong and a 8 week structured Line dancing for the research study on cancer survivors.

\subsection{The Objectives of the Study}

In order to examine the final aim of the study on whether Qi-Gong is a better form of physical activity than other form, a structured program was developed with expert from the field.

To present the content and gradual progression of a 8 weeks physical activity in line with the level of fatigue and distress build up in breast cancer survivors.

\section{Theoretical Basis to Study}

Theoretical background for this study will be drawn from Bandura's Social Cognition Theory. According to [26], self-efficacy has been linked to PA participation. In social cognitive theory, self-efficacy is a central influence on exercise behavior [27] and is a promising theoretical framework for understanding and promoting PA behaveior in breast cancer patients [28-30]. This proposition is 
supported by the results of a number of studies of adults in which efficacy based programs significantly influenced exercise adherence [31]. High self-efficacy motivates behavior. In turn, successful enactment or performance of a behavior resulting in pleasurable sensations further enhances self-efficacy in a particular domain [32]. Most studies reviews that perceived barriers [33] selfregulatory behavior [34] and outcome expectancies [35] are the constructs that have indirect effect of self efficacy on PA.

\section{Methodology}

\subsection{Study Design}

Ethical approval for the entire study was obtained from The Malaysia Ministry of Health Ethical Committee and the UMMC (University Malaya Medical Centre) Medical Ethics Committee.

\subsection{Setting}

The research study will be conducted at University Malaya, medical centre located centrally in Malaysia, once the protocol are developed. It has a specialized breast clinic which is partially funded by the government and it receives referrals from all over the country. It also has a private wing, the Specialist Centre, where fee-paying patients can expect a shorter waiting list for medical treatment.

\subsection{Recruitment}

Women diagnosed with stage 1-II breast cancer from around the region will be sent a letter by their breast surgeon inviting them to the study. A research coordinator and the primary investigator discussed the needs of the women and the physical activity at moderate intensity for building the cancer survivors. The program will be developed and patients who consented on the telephone contact, will receive an information sheet, consent form and questionnaire related to the phases of studies. Participants will be asked to get a letter of fitness that they can engage in physical activity and their BP will be monitored.

\section{Inclusion and Exclusion Criteria}

The participants selected will be based on the following eligibility criteria:

1) 18 - 65 years;

2) First time diagnosis of breast cancer with no metastasis;

3) Stage I-II breast cancer;

4) At least 1 year post diagnosis.

The exclusion criteria are:

1) Medical contraindications of exercise (e.g. epilepsy, uncontrolled hypertension, significant orthopedic prob- lem or cardiovascular disease);

2) Currently participating/practicing Qi-Gong or aerobic exercise;

3) Already engaging in more than 4 hours of active activities per week.

The rationale of having women preferably after active treatment is because this is a period where active support are withdrawn since treatment have ended, and thus present a period for women to reenact healthy changes to their live-styles, and for therapists to focus on enabling lifestyle rebuilding. These women need to rebuild their lives and maintain an active lifestyle.

\subsection{Power Calculation and Sample Size}

Sample size for the research trial will be calculated for a between-group mean difference of 10 units SD (standard division) on the FACT-G score; with at least 38 participants in each group - the trial will have $80 \%$ power at $\mathrm{p}$ $<0.05$ [36].

\subsection{Instruments}

FACT-B is a 44-item self report on multidimensional health related QOL. It consists of FACT-G aspects: physical well-being (PWB), functional well-being (FWB), emotional well-being (EWB), social/family well-being (SWB) and an additional part of Breast Cancer Subscale (BCS). The alpha coefficient (internal consistency) for the FACT-B total score has been reported as 0.90 , with subscale alpha coefficients ranging from 0.63 to 0.86 [37]. Health-related quality of life is an essential outcome measurement in cancer clinical trials.

The Depression, Anxiety, Stress Scale (DASS-21) allows participants to self-report the degree of negative emotional symptoms experienced during the previous week on 21-items, which can be reliably grouped as three scales (Anxiety, Stress and Depression). Alpha values for the 7-item scales range from 0.73 (Anxiety) to 0.81 (Stress and Depression) [38].

Bandura's Exercise Self efficacy rated one's adherence to exercise based on a 100-point percentage scale which comprised of 10-point increments, ranging from $0 \%$ (not at all confident) to $100 \%$ (highly confident). The score is derived by averaging the overall score and is directly proportional to the adherence of an exercise activity. This 18-item scale has been reported to have a single factor structure with high internal consistency of 0.95 , with no floor or ceiling effect [39].

FACT-F composes 41 items which included 28 items from FACT-G and extra 13 items related to fatigue for the past 7 days. This self reported tool found to be stable with test-retest $r=0.87$ and internally consistent (coefficient alpha range $=0.95-0.96$ ). The fatigue subscale has an alpha range from $0.93-0.95$ [40]. 


\subsection{Independent Variables}

The Experimental Group-This is the 8-week Qi-Gong intervention group: Participants in the experimental group received the intervention along the usual standard medical care. The intervention will be a 8 -week course of about 1.5 hours per week/session. Heart rate and ratings of perceived exertion will be assessed using Borg scale. A video compact disc with Qi-Gong steps will be recorded and given to the group members as the follow-up program at home. Each subjects needs to carry out the program at home for 30 minutes 3 - 5 times a week. The progress will be recorded in a log book. Buddy system (where a participant is assigned another participant and they are assigned responsibilities to each others) will be inbuilt as the support mechanism to promote collaborative support between participants.

The 8-week line dancing (placebo) - This line dancing program will consist of about 90 minutes with warm up period, exercise sequences and a cool down period. The subjects in this group will meet the instructor once a week for 90 minutes. Heart rate and ratings of perceived exertion will be assessed using Microlife BP sets and Borg scale. For the follow-up home program, each subject will be given a video compact disc with the line dancing steps recorded and is required to carry out it at home. Every participant will need to practice 3 - 5 times of line-dancing class, 30 minutes per session in a week. A log book will be given to monitor behavior adherence on the program. Buddy-support system will be introduced as well.

The Usual Care Group (Control) - This is the usualcare group: Participants in this comparative group received only the usual standard medical care. As the use of a no-treatment for the control group may be unacceptable to patients, a waiting list where all control participants would ultimately receive the intervention will be offered after the research study ended.

\subsection{Data Collection Scheme}

Three measurements will be taken: baseline/pretest (T1), post-intervention/post-test (T2).

Post-intervention measure will be taken immediately after the 8-week program. They will be then given the baseline questionnaire package to fill up. Working women will be provided a letter to their employer requesting support for time-off during the sessions, and those requiring half time slip will be provided. To ensure participation, interested participants, unable to get support from their employer, will be given time-off certificate or medical leave certificates by the attending breast surgeon. In the control group, patients will be identified from the breast cancer database, and those who consented will be asked to come in to the medical centre.

\section{Data Analysis}

The statistical analyses for the research study will be performed using the Statistical Package for Social Sciences, version 16 [41]. The demographical and clinical characteristic of the sample will be described using descriptive statistics, and where appropriate, the two groups will be compared using either Pearson Chi-Square $\left(\mathrm{X}^{2}\right)$ for categorical variables or independent-samples T-tests /ANOVA for continuous variables. The nonparametric Wilcoxon one-sample signed rank test (a nonparametric alternative to the one-sample or paired samples parametric $t$-test) will be conducted to test changes over time 1 (pretest) and time 2 (posttest) if the distribution is not normal. The covariates would be the baseline measures of the endpoints, and the demographic variables found significant at baseline. Paired sample $t$ tests will examine if there is a change in subject's QOL from time 1 to time 2). Two way (group $\times$ time) repeated measures ANOVA will examine differences between groups over time. All tests will be two-tailed and an alpha level of 0.05 will be applied as the criterion for statistical significance.

\section{Result}

The results of the preplanning with the experts (the Qi-Gong masters and the Line dancing gurus) lead to the development of the 8 weeks structured Qi-Gong (see Appendix 2) and the development of the 8 weeks placebo, Line dancing (see Appendix 1) the final research study would lead to the understanding on how to engage cancer survivors to participate in an active lifestyle and if the intervention improves QOL and reduce distress. The 8 weeks protocol for Qi-Gong (Appendix 2) and the 8 week protocol for Line dancing outline the sequential steps needed to build the physical health in a group format so that the cancer survivors can come together and support each other via modeling, social persuasion and mastery. This builds up their self efficacy to engage in health behavior. Thus the protocol were designed informed by the Social Bandura Theory to ensure successful health behaviours.

\section{Conclusions}

The developed protocol could be used by researchers to replicate study so that the findings are comparable. Healthcare professionals such as Occupational Therapists with their expertise in occupational participation and lifestyle redesign should collaborate with the culturally popular groups like Qi-Gong associations, to design suitable cancer survivorship program so that they can assist with the rehabilitation and prevention aspects of an increasing number of cancer survivors all over the world. 
The implication of such activity intervention is that it benefits both patients and activity-provider, and could help reduce healthcare costs associated with the human resources and long-term effects of cancer patients living in the community.

\section{Acknowledgements}

The authors wish to thank everyone who have contributed to the study: especially the Qi Neng Qi-Gong who provided the exercise regimes, and the women from UMMC and Breast Welfare Association who participated in the study. This study is sponsored by University Malaya Research grant (UMRG 32/2006).

\section{REFERENCES}

[1] American Cancer Society, "Breast Cancer Facts and Figures," ACS, Atlanta, 2005.

[2] A. Bandura, "Social Foundations of Thought and Action: A Social Cognitive Theory," Prentice-Hall, Englewood Cliffs, 1986.

[3] M. J. Brady, D. F. Cella, F. Mo, A. E. Bonomi, D. S. Tulsky, S. R. Lloyd, S. Deasy, M. Cobleigh and G. Shiomoto, "Reliability and Validity of the Functional Assessment of Cancer Therapy-Breast Quality-of-Life Instrument," Journal of Clinical Oncology, Vol. 15, No. 3, 1997, pp. 974-986.

[4] M. Cardol, R. De Haan, B. De Jong, G. Van Den Bos and I. De Groot, "Impact on Participation and Autonomy (IPA)," Archives of Physical Medicine and Rehabilitation, Vol. 82, No. 2, 2001, pp. 210-216. doi:10.1053/apmr.2001.18218

[5] Centers for Disease Control and Prevention, "Physical Activity for Everyone," 2010. http://www.cdc.gov/physicalactivity/everyone/guidlines/i ndex.html

[6] K. Chen and R. Yeung, "Exploratory Studies of Qi-Gong Therapy for Cancer in China," Integrative Cancer Therapies, Vol. 1, No. 4, 2002, pp. 345-370. doi:10.1177/1534735402238187

[7] L. Clegg, F. Li, B. Hankey, K. Chu and B. K. Edwards, "Cancer Survival among US Whites and Minorities: SEER (Surveillance, Epidemiology, and End Results) Program Population-Based Study," Archives of Internal Medicine, Vol. 162, 2002.

[8] M. Cohen, "Towards a Framework for Women's Health," Patient Education and Counseling, Vol. 33, No. 3, 1998, pp. 187-196. doi:10.1016/S0738-3991(98)00018-4

[9] J. Corbin and A. Strauss, "Unending Work and Care: Managing Chronic Illness at Home," Jossey-Bass Publishers, San Francisco, 1988.

[10] K. S. Courneya, "Exercise in Cancer Survivors: An Overview of Research," Medicine \& Science in Sports \& Exercise, Vol. 35, No. 11, 2003, pp. 1846-1852. doi:10.1249/01.MSS.0000093622.41587.B6
[11] K. S. Courneya, "Exercise and Quality of Life in Cancer Survivors," In: G. Faulkner and A. Taylor, Eds., Exercise, Health and Mental Health: Emerging Relationships, Routledge, Abingdon, 2005.

[12] K. S. Courneya, J. R. Mackey and D. C. McKenzie, "Exercise for Breast Cancer Survivors: Research Evidence and Clinical Guidelines," Physician and Sports Medicine, Vol. 30, No. 8, 2002, pp. 33-42. doi: $10.3810 / \mathrm{psm} .2002 .08 .402$

[13] A. J. Daley, H. Crank, J. M. Saxton, N. Mutrie, R. Coleman and A. Roalfe, "Randomized Trial of Exercise Therapy in Women Treated for Breast Cancer," Journal of Clinical Oncology, Vol. 25, No. 13, 2007, pp. 17131721. doi:10.1200/JCO.2006.09.5083

[14] R. K. Dishman, R. W. Motl, J. F. Sallis, A. L. Dunn, A. S. Birnbaum, G. J. Welk, et al., "Self Management Strategies Mediate Self-efficacy and Physical Activity," American Journal of Preventive Medicine, Vol. 29, No. 1, 2005, pp. 10-18. doi:10.1016/j.amepre.2005.03.012

[15] V. Erickson, M. Pearson, A. Patricia, J. Adams and K. Kahn, "Arm Edema in Breast Cancer Patients," Journal of the National Cancer Institute, Vol. 93, No. 2, 2001, pp. 96-111. doi:10.1093/jnci/93.2.96

[16] B. Everett, Y. Salamonson and P. M. Davidson, "Bandura's Exercise Self-Efficacy Scale: Validation in an Australian Cardiac Rehabilitation Setting," International Journal of Nursing Studies, Vol. 46, No. 6, 2009, pp. 824829. doi:10.1016/j.ijnurstu.2009.01.016

[17] A. Faulkner, "Strategies for Living: A Report of User-Led Research into People's Strategies for Living with Mental Distress," Mental Health Foundation, London, 2000.

[18] C. M. Friedenreich and A. E. Cust, "Physical Activity and Breast Cancer Risk: Impact of Timing, Type and Dose of Activity and Population Subgroup Effects," British Journal of Sports Medicine, Vol. 42, No. 8, 2008, pp. 636-647. doi:10.1136/bjsm.2006.029132

[19] D. A. Galvão and R. U. Newton, "Review of Exercise Intervention Studies in Cancer Patients," Journal of Clinical Oncology, Vol. 23, No. 4, 2005, pp. 899-909. doi:10.1200/JCO.2005.06.085

[20] P. Ganz and E. Hahn, "Implementing a Survivorship Care Plan for Patients with Breast Cancer," Journal of Clinical Oncology, Vol. 26, No. 5, 2008, pp. 759-767. doi:10.1200/JCO.2007.14.2851

[21] C. Gore-Felton and D. Spiegel, “Enhancing Women's Lives: The Role of Support Groups among Breast Cancer Patients," The Journal for Specialists in Group Work, Vol. 24, No. 3, 1999, pp. 274-287. doi:10.1080/01933929908411436

[22] M. D. Holmes, W. Y. Chen, D. Feskanich, et al., "Physical Activity and Survival after Breast Cancer Diagnosis," Journal of American Medical Association, Vol. 293, No. 20, 2005, pp. 2479-2486. doi:10.1001/jama.293.20.2479

[23] R. Knols, N. K. Aaronson, D. Uebelhart, J. Fransen and G. Aufdemkampe, "Physical Exercise in Cancer Patients during and after Medical Treatment: A Systematic Re- 
view of Randomized and Controlled Clinical Trials," Journal of Clinical Oncology, Vol. 23, No. 16, 2005, pp. 3830-3842. doi: $10.1200 /$ JCO.2005.02.148

[24] B. R. Lorraine, J. P. Nola, L. R. David, S. K. Anamaria and B. P. Monika, "Research in Nursing \& Health," Physical Activity, Self-Efficacy, and Perceived Exertion among Adolescents, Vol. 27, No. 6, 2004, pp. 435-446.

[25] S. Lovibond and P. Lovibond, "Manual for the Depression Anxiety Stress Scales," 2nd Edition, Psychology Foundation, Sydney, 1995.

[26] G. Maskarinec, S. Murphy, D. M. Shumay and H. Kakai, "Dietary Changes among Cancer Survivors," European Journal of Cancer Care, Vol. 10, No. 1, 2001, pp. 12-20. doi:10.1046/j.1365-2354.2001.00245.x

[27] E. McAuley, "The Role of Efficacy Cognitions in the Prediction of Exercise Behavior in Middleaged Adults," Journal of Behavioral Medicine, Vol. 15, No. 1, 1992, pp. 6588. doi:10.1007/BF00848378

[28] E. McAuley and B. Blissmer, "Self-Efficacy Determinants and Consequences of Physical Activity," Exercise and Sport Sciences Reviews, Vol. 28, No. 2, 2000, pp. 85-88.

[29] A. McTiernan, "Physical Activity after Cancer: Physiologic Outcomes," Cancer Invest, Vol. 22, No. 1, 2004, pp. 68-81. doi:10.1081/CNV-120027581

[30] National Cancer Registry, "The Second Report of the National Cancer Registry-Cancer incidence in Malaysia," National Cancer Registry, Ministry of Health, Kuala Lumpur, 2003.

[31] NHMRC, "Psychosocial Clinical Practice Guidelines: Information, Support and Counseling for Women with Breast Cancer," Commonwealth of Australia, Canberra, 2000.

[32] B. Oh, P. Butow, S. Clarke, P. Beale, N. Pavlakis, E. Kothe, et al., "Impact of Medical Qi-Gong on Quality of Life, Fatigue, Mood and Inflammation in Cancer Patients: A Randomized Controlled Trial," Annals of Oncology, Vol. 21, No. 3, 2010, pp. 608-614. doi:10.1093/annonc/mdp479

[33] R. L. Petosa, R. Suminski and B. Hortz, "Predicting Vigorous Physical Activity Using Social Cognitive Theory," American Journal of Health Behaviors, Vol. 27, No. 4,
2003, pp. 301-310.

[34] B. M. Pinto, N. C. Maruyama, M. M. Clark, D. G. Cruess, E. Park and M. Roberts, "Motivation to Modify Lifestyle Risk Behaviors in Women Treated for Breast Cancer," Mayo Clinic Proceedings, Vol. 77, No. 2, 2002, pp. 122 129. doi: $10.4065 / 77.2 .122$

[35] L. Q. Rogers, C. Matevey, P. Hopkins-Price, P. Shah, G. Dunnington, and K. S. Courneya, "Exploring Social Cognitive Theory Constructs for Promoting Exercise among Breast Cancer Patients," Cancer Nursing, Vol. 27, No. 6, 2004, pp. 462-473. doi:10.1097/00002820-200411000-00006

[36] L. Q. Rogers, P. Shah, G. Dunnington, A. Greive, A. Shanmughan, B. Dawson, et al., "Social Cognitive Theory and Physical Activity during Breast Cancer Treatment," Oncology Nursing Forum, Vol. 32, No. 4, 2005, pp. 807-815. doi:10.1188/05.ONF.807-815

[37] L. S. Rovniak, E. S. Anderson,, R. A. Winett and R. S. Stephens, "Social Cognitive Determinants of Physical Activity in Young Adults: A Prospective Structural Equation Analysis," Annals of Behavioral Medicine, Vol. 24, No. 2, 2002, pp. 149-157. doi:10.1207/S15324796ABM2402_12

[38] SPSS, "SPSS 16.0 Brief Guide (Vol. 1)," Prentice Hall, Chicago, 2007.

[39] J. K. Vallance, K. S. Courneya, L. M. Taylor, R. C. Plotnikoff and J. R. Mackey, "Development and Evaluation of a Theory-Based Physical Activity Guidebook for Breast Cancer Survivors," Health Education Behaviour, Vol. 35, No. 2, 2008, pp. 174-189. doi:10.1177/1090198106287693

[40] WHO, "World Health Organization. Innovative Care for Chronic Conditions: Building Blocks for Action: Global Report," Patient Education and Counseling, 2002. www.who.int/chronic_conditions/en/icccglobalreport.pdf

[41] S. B. Yellen, D. F. Cella, K. Webster, C. Blendowski and E. Kaplan, "Measuring Fatigue and Other Anemia-Related Symptoms with the Functional Assessment of Cancer Therapy (FACT) Measurement System," Journal of Pain and Symptom Management, Vol. 13, No. 2, 1997, pp. 63-74. doi:10.1016/S0885-3924(96)00274-6 


\section{Appendix 1}

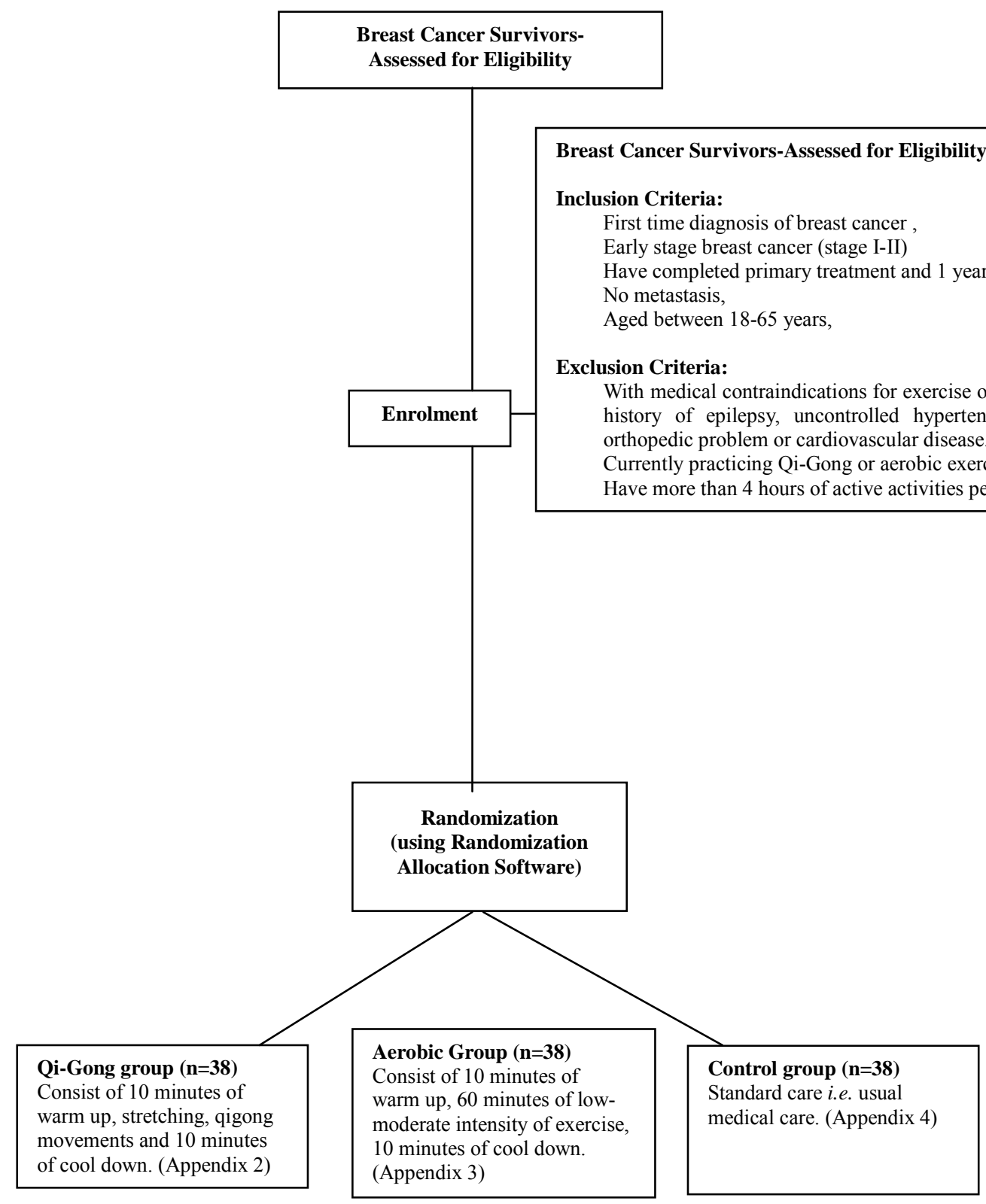

\section{Appendix 2. 8 Weeks Protocol for Qi-Gong Group}

Objective: To evaluate quality of life of breast cancer survivors based on 2 common physical activities which are Qi-Gong and aerobic exercise.

Duration: 8 weeks (once a week face-to-face session, at least 2 times at home with self recorded log).

Procedure: Patients will be recruited at UMMC via telephone invitation.

After that, patients will be screened for eligibility based on inclusion and exclusion criteria.

Eligible patients will sign consent form and random- ized into 3 groups (Qi-Gong, Aerobic and control).

Patients will be called back for sessions in the group where they are randomized and as scheduled.

The description of each session is as below:

\section{SESSION 1 Qi-Gong}

Fill up questionnaires: Demographic background, FACTB, DASS 21, ESS, FACT-F, introduced buddy system and $\log$ book will be distributed.

Get to know each other, assigning buddy based on house location.

Measure blood pressure and heart rate, check if there is anyone not feeling well. 


\section{Activity Session: 90 minutes}

I. Warming up (10 minutes)

- Introduction to Qi-Gong and its benefit

- Introduction to Peng Qi Guan Ding Fa

II. Main exercise (60 minutes)

- Preparatory Posture

- Starting and ending sequence

- Minor Qi-Gong Practices-Kai he La Qi Fa

- 2 interval of 5 minutes rest in between

III. Cool down (10 minutes)

- Remind for next session's date and the use of the log book

\section{SESSION 2}

Discuss for assigned home program.

Measure blood pressure and heart rate, check if there is anyone not feeling well.

\section{Activity Session: 90 minutes}

I. Warming up (10 minutes)

- Revision of lesson learned

II. Main exercise (60 minutes)

- Peng Qi Guan Ding Fa-Starting sequence, Sequence One

- Front starting, side lifting, and concluding sequence.

- Minor Qi-Gong Practices-Kai He La Qi Fa and Chen Qi Fa

- 2 interval of 5 minutes rest in between

III. Cool down (10 minutes)

- Remind for next session's date and the useof the log book

SESSION 3 Qi-Gong

\section{Activity Session: 90 minutes}

I. Warming up (10 minutes)

- Revision of previous lesson learned.

II. Main exercise (60 minutes)

- Continuation of Peng Qi Guan Ding Fa. Peng Qi Guan Ding Fa-Starting sequence, Sequence One

- Sequence Two: Sides starting and Front Lifting and

- Sequence Three : Diagonal starting and Lifting.

- Minor Qi-Gong Practices - Kai he La Qi Fa and Chen Qi Fa

- 2 interval of 5 minutes rest in between.

III. Cool down (10 minutes)

[Remind for next session's date and the use of the log book (10 minutes)].

\section{SESSION 4 Qi-Gong}

Discuss for assigned home program.

Measure blood pressure and heart rate, check if there is anyone not feeling well.

\section{Activity Session: 90 minutes}

I. Warming up (10 minutes)

- Revision of previous lesson learned.

II. Main exercise (60 minutes)

- Practice Peng Qi Guan Ding Fa using oral Instruction $\mathrm{CD}$

- Minor Qi-Gong Practices-Dun Qiang Fa

- 2 interval of 5 minutes rest in between

III. Cool down (10 minutes)

[Remind for next session's date and the use of the log book].

\section{SESSION 5 Qi-Gong}

Discuss for assigned home program.

Measure blood pressure and heart rate, check if there is anyone not feeling well.

\section{Activity Session: 90 minutes}

I. Warming up (10 minutes)

- Revision of previous lesson learned

- Warming up exercises using Kai He La Qi and Chen Qi

II. Main exercise (60 minutes)

- Practice Peng Qi Guan Ding from beginning to the end

- Minor Qi-Gong Practice- Dun Qiang Fa

- 2 interval of 5 minutes rest in between

III. Cool down (10 minutes)

[Remind for next session's date and the use of the log book].

\section{SESSION 6 Qi-Gong}

Discuss for assigned home program.

Measure blood pressure and heart rate, check if there is anyone not feeling well.

\section{Activity Session: 90 minutes}

I. Warming up (10 minutes)

- Revision of previous lesson learned

II. Main exercise (60 minutes)

- Practice Peng Qi Guan Ding Fa. Instructors will reinforce learning by explaining finer details of movement and how to use the mind to gather qi

- San Xin Bing Zhan Zhuang

- 2 interval of 5 minutes rest in between

III. Cool down (10 minutes)

Remind for next session's date and the use of the log book.

\section{SESSION 7 Qi-Gong}

Discuss for assigned home program.

Measure blood pressure and heart rate, check if there is anyone not feeling well.

\section{Activity Session: 90 minutes}

I. Warming up (10 minutes) 
Revision of previous lesson learned

II. Main exercise (60 minutes)

- Kai He La Qi and Chen Qi

- Practise Peng Qi Guan Ding and San Xin Bing Zhan Zhuang

- 2 interval of 5 minutes rest in between

III. Cool down (10 minutes)

[Adherence Strategy: Reminder for next session's date and the use of the log book]

SESSION 8 Qi-Gong

Discuss for assigned home program.

Measure blood pressure and heart rate, check if there is anyone not feeling well.

\section{Activity Session: 90 minutes}

I. Warming up (10 minutes)

- Revision of previous lesson learned

II. Main exercise (60 minutes)

- General revisions

- Practice Peng Qi Guan Ding and San Xin Bing Zhan Zhuang

- 2 interval of 5 minutes rest in between

III. Cool down (10 minutes)

- Reevaluation- fill up questionnaires: FACT-B, DASS 21, FACT-F

- Ending for the 8-week program, log book will be collected from each patient

[All data will be collected, coded, documented and kept away in locked cupboard].

\section{Appendix 3. 8 weeks Protocol for Line Dance Group}

Objective: To evaluate quality of life of breast cancer survivors based on 2 common physical activities which are Qi-Gong and aerobic exercise.

Duration: 8 weeks

Procedure:

1) Patients will be recruited at UMMC via telephone invitation.

2) After that, patients will be screened for eligibility based on inclusion and exclusion criteria.

3) Eligible patients will sign consent form and randomized into 3 groups (Qi-Gong, Aerobic and control).

4) Patients will be called back for sessions in the group where they are randomized and as scheduled.

5) The description of each session is as below:

Session 1 LDG

Fill up questionnaires: Demographic background, FACTB, DASS 21, ESS, FACT-F, introduce buddy system and log book will be distributed.

Get to know each other, assigning buddy based on house location.

Measure blood pressure and heart rate, check if there is anyone not feeling well.

\section{PA Session: 90 minutes}

I. Warming up (10 minutes)

- Introduction to Line Dance

- Stretching exercise

II. Main exercise (60 minutes)

- Marcarena steps will be taught

- Dance with music

- 2 interval of 5 minutes rest in between

III. Cool down (10 minutes)

Reminded regarding home exercise and use of the log book.

Session 2 LDG

Discuss for assigned home program.

Measure blood pressure and heart rate, check if there is anyone not feeling well.

\section{PA Session}

I. Warming up (10 minutes)

- Stretching exercise

II. Main exercise (60 minutes)

- Revision of previous lesson

- Achy Breaking Heart steps

- Practice with music

- 2 interval of 5 minutes rest in between

III. Cool down (10 minutes)

Remind for next session's date and the use of the log book.

\section{Session 3 LDG}

Discuss for assigned home program.

Measure blood pressure and heart rate, check if there is anyone not feeling well.

I. Warming up (10 minutes)

- Stretching Exercise

II. Main exercise (60 minutes)

- Revision of previous lesson learned- Macarena and Achy Breaking Heart

- 2 interval of 5 minutes rest in between

III. Cool down (10 minutes)

Remind for next session's date and the use of the log book.

Session 4 LDG

Discuss for assigned home program.

Measure blood pressure and heart rate, check if there is anyone not feeling well.

\section{PA Session}

I. Warming up (10 minutes)

- Stretching Exercise

II. Main exercise (60 minutes)

- Revision of previous lesson learned

- Saturday Night Fever steps 


\author{
- 2 interval of 5 minutes rest in between \\ III. Cool down (10 minutes) \\ Remind for next session's date and the use of the log
} book.

\section{Session 5 LDG}

Discuss for assigned home program.

Measure blood pressure and heart rate, check if there is anyone not feeling well.

PA session

I. Warming up (10 minutes)

- Stretching Exercise

II. Main exercise (60 minutes)

- Revision of previous lesson learned

- Saturday Night Fever-Dance with music

- 2 interval of 5 minutes rest in between

III. Cool down (10 minutes)

Remind next session's date and the log book.

\section{Session 6 LDG}

Discuss for assigned home program.

Measure blood pressure and heart rate, check if there is anyone not feeling well.

\section{PA Session}

I. Warming up (10 minutes)

- Stretching Exercise

II. Main exercise (60 minutes)

- Revision of previous lesson learned

- Let your Love flow steps

- 2 interval of 5 minutes rest in between

III. Cool down (10 minutes)

Remind for next session's date and log book.

Session 7 LDG
Discuss for assigned home program.

Measure blood pressure and heart rate, check if there is anyone not feeling well.

\section{PA Session}

I. Warming up (10 minutes)

- Stretching Exercise

II. Main exercise (60 minutes)

- Revision of previous lesson learned

- Let your Love Flow: Dance with music

- 2 interval of 5 minutes rest in between

III. Cool down (10 minutes)

Remind for next session's date and the log book.

Session 8 LDG

Discuss for assigned home program.

Measure blood pressure and heart rate, check if there is anyone not feeling well.

\section{PA Session}

I. Warming up (10 minutes)

- Stretching Exercise

II. Main exercise (60 minutes)

- Revision of previous lesson learned

- General revisions of all dances that learnt

- 2 interval of 5 minutes rest in between

III. Cool down (10 minutes)

Reevaluation- filled up questionnaires:

FACT-B, DASS 21, FACT F

Ending for the 8-week program, log book will be collected from each patient.

[All the data will be collected, coded, documented and kept away in locked cupboard]. 